\title{
Effects of refined Glycyrrhiza uralensis polysaccharide on intestinal flora and intestinal barrier function in broilers: High-throughput sequencing study
}

\author{
X R. Liang ${ }^{1}$, K X. Lian ${ }^{1}$, D Y. Wang ${ }^{1}$, B J. Zhang ${ }^{1}$, Y J. Qiao ${ }^{1}$, L. Yang ${ }^{1}$, M M. Wang ${ }^{1}$, \\ Y B. Shao ${ }^{1}, \mathrm{X} \mathrm{L.} \mathrm{Gu}{ }^{1 *}$ and Y. Luo ${ }^{1 *}$
}

College of Animal Science and Technology, Shihezi University, Xinjiang - 832000, People's Republic of China

\begin{abstract}
This study was conducted to investigate the effects of Glycyrrhiza uralensis polysaccharide (GUP) on intestinal flora and intestinal barrier function of $E$. coli 078 -induced intestinal damage in broilers. A total of 120 one-day-old male broilers with similar initial body weights were obtained from a local breeding company and used in this study. Broilers were randomly allocated to 5 treatments. The 5 treatment groups were as follows: 1) control group (broilers given a basal diet); 2) Escherichia coli 078-challenged group (broilers given a basal diet and receiving intraperitoneal administration of Escherichia col O78); 3) high-dose GUP group (broilers given a basal diet supplemented with $15 \mathrm{mg} / \mathrm{kg}$ GUP gavage and receiving intraperitoneal administration of Escherichia coli O78); 4) medial-dose GUP group (broilers given a basal diet supplemented with $10 \mathrm{mg} / \mathrm{kg}$ GUP gavage and receiving intraperitoneal administration of Escherichia coli O78); and 5) low-dose GUP group (broilers given a basal diet supplemented with $5 \mathrm{mg} / \mathrm{kg}$ GUP gavage and receiving intraperitoneal administration of Escherichia coli $\mathbf{0 7 8}$ ). Intestinal flora composition and detection of intestinal barrier function were examined by intestinal histopathology analysis, ELISA analysis, 16S rDNA high-throughput sequencing and quantitative real-time PCR (RT-PCR). The villous structure of the intestine was observed by intestinal histopathology analysis. The contents of D-lactic acid (D-LA), diamine oxidase (DAO) and endotoxin in plasma were detected by ELISA. mRNA expression levels of the tight junction (TJ) proteins were measured using RT-PCR. Our results suggested that the intestinal walls of the model group were damaged. The villi were gradually restored after GUP treatment. ELISA showed that the levels of plasma endotoxin, D-LA and DAO decreased in the GUP groups compared to those in the model group. GUP treatment also increased the mRNA expression of ZO-1, occludin and claudin-1. High-throughput sequencing of 16S rDNA showed that GUP increased the diversity of intestinal flora, changed the microbial composition and increased the Bacteroidetes/Firmicutes ratio. In conclusion, GUP treatment could improve the fecal microbiota composition and repair intestinal barrier function. The study suggested that GUP may be used as a therapy for the treatment or prevention of intestinal disease.
\end{abstract}

Key words: Broiler, Glycyrrhiza uralensis polysaccharide, Intestinal barrier function, Intestinal flora

\section{INTRODUCTION}

Balance of intestinal flora and integrity of intestinal barrier function together promote host health. Intestinal flora are not only involved in the digestion of food and stimulate the immune system but are also one of the three major components of the intestinal barrier (the symbiotic bacteria that inhabit the bacterial surface, the epithelium formed by epithelial cells and the intercellular connections and intestinalassociated lymphoid tissue, including various immune cells) (Cukrowska et al., 2017; Xue et al., 2018). Intestinal flora can affect intestinal permeability by regulating the fermentation and absorption of polysaccharides in the feed. Accordingly, intestinal barrier dysfunction leads to rampant invasion of pathogenic bacteria, and the synergistic effect of the two causes further deterioration of intestinal diseases (Xiao et al., 2018). Intestinal epithelial barrier homeostasis is regulated by tight junction (TJ) proteins, such as ZO-1, occludin, and claudin-1, which play

"Corresponding Author, E Mail: luoyan@shzu.edu.cn, xlgu@shzu.edu.cn 
vital roles in maintaining barrier function and protecting the body from intestinal allergens, toxins and pathogens. Recent studies have shown that a variety of intestinal diseases are related to intestinal flora disorders and are associated with intestinal mucosal barrier dysfunction (Wang et al., 2014; Ping et al., 2015). Therefore, regulating the balance of intestinal flora or restoring the balance of the intestinal epithelial barrier internal environment should be the most urgent and feasible strategies to inhibit the occurrence of intestinal diseases. Many studies have been conducted to identify effective natural drug therapies that can cure or prevent intestinal diseases by targeting an immature intestinal barrier and intestinal flora imbalance.

Pathogenic Escherichia coli infection can destroy the structure of intestinal flora and intestinal mucosa, leading to decreased production performance and increased mortality in poultry. Among the pathogenic Escherichia coli in birds, serotype $\mathrm{O} 78$ is the main strain causing Escherichia coli infection (Wang et al., 2017). Its invasion not only affects the balance of intestinal flora but also produces endotoxin, which causes intestinal mucosal damage, increases intestinal permeability, and induces endotoxemia, systemic inflammatory response and multiorgan system dysfunction syndrome (Yongjun, 2008; Bian et al., 2016;). Clinical symptoms include septicemia, synovitis, peritonitis, granuloma, salpingitis, balloon disease, and pulse inflammation. Although clinical poultry Escherichia coli disease can occur on its own, it can also occur with other bacterial or viral diseases mixed together making it difficult to diagnose and treat; in addition, clinical poultry Escherichia coli disease is a harm to the poultry industry and an important disease to the poultry industry, causing huge economic losses.

As a result, research on treatments derived primarily from plant-based medicines such as herbs and spices is attracting more interest because these supplements are natural, perform many beneficial functions, have minimal side effects and have no resistance or residue (Yang et al., 2019). In recent years, studies have shown that Chinese medicine polysaccharides regulate and protect the intestinal flora and the intestinal mucosal barrier function (Wang et al., 2015). For example, ginseng neutral polysaccharides can promote the repair of intestinal structures in AAD mice, regulate the composition and diversity of intestinal flora in mice, and achieve the role of rebuilding intestinal microecology (Qi et al., 2019). The polysaccharides of Radix Codonopsis can promote growth, regulate the balance of intestinal microecology and improve immune function (Li et al., 2017). The total polysaccharides in Sijunzi decoction can reduce the barrier function of intestinal epithelial cells induced by tumor necrosis factor-kappa B (Yue et al., 2018). Dendrobium candidum polysaccharide can protect the intestinal mucosal barrier by reducing the loss of zonula occludens-1 (ZO-1) and occludin in adjacent tissues (Jian et al., 2019). Glycyrrhiza polysaccharide can fight inflammation (Zhao et al., 2015) and oxidation (Liao et al., 2015), has antisepsis and antivirus (Lirong et al., 2011), and tumor-fighting abilities (Li et al., 2016), and can improve immune function and inhibit bovine AIDS virus, adenovirus type (AdV) and coxsackievirus (CBV3) (Zhu et al., 2018). However, the effect of GUP on intestinal flora and intestinal barrier function of intestinal impaired broilers is unclear. In this study, $16 \mathrm{~S}$ rDNA high-throughput sequencing technology was used to study the regulation of intestinal flora in broilers with intestinal damage induced by E. coli $\mathrm{O} 78$ after Glycyrrhiza uralensis polysaccharide (GUP) gavage. The effect of polysaccharides on intestinal barrier function was also observed.

\section{MATERIALS AND METHODS}

Glycyrrhiza uralensis polysaccharide (GUP) was extracted from Shihezi University Chinese Traditional Veterinary Laboratory (Lian et al., 2018).

The Escherichia coli 078 standard strain 
was placed in a nutrient broth at $37^{\circ} \mathrm{C}$ for $30 \mathrm{~h}$, and a small amount of inoculation was removed for expansion. A bacterial plate (LB medium) was placed at $37^{\circ} \mathrm{C}$ for $24 \mathrm{~h}$ under aerobic conditions, and the concentration of the solution was determined. The concentration was adjusted to $1.0 \times 10^{9} \mathrm{CFU} / \mathrm{mL}$. The pretest showed that the challenge dose was $1.0 \times 10^{6}$ $\mathrm{CFU} / \mathrm{mL}$, and the reconstituted E. coli $\mathrm{O} 78$ standard strain was diluted to $1.0 \times 10^{6} \mathrm{CFU} / \mathrm{mL}$; the average dosage was $1 \mathrm{~mL}$ per chicken for 14-16 days (Yongjun, 2008; Hou et al., 2018).

The animal use experimental procedures involve in this study were approved by the Animal Care and Use Committee of Shihezi University. A total of 120 one-day-old male broilers with similar initial body weights were obtained from a local breeding company and used in this study. Broilers were randomly allocated to 5 treatments. The 5 treatment groups were as follows: 1) control group (broilers given a basal diet); 2) Escherichia coli O78-challenged group (broilers given a basal diet and receiving intraperitoneal administration of Escherichia coli O78); 3) high-dose GUP group (broilers given a basal diet supplemented with $15 \mathrm{mg} / \mathrm{kg}$ GUP gavage and receiving intraperitoneal administration of Escherichia coli O78); 4) medial-dose GUP group (broilers given a basal diet supplemented with $10 \mathrm{mg} / \mathrm{kg}$ GUP gavage and receiving intraperitoneal administration of Escherichia coli O78); and 5) low-dose GUP group (broilers given a basal diet supplemented with $5 \mathrm{mg} / \mathrm{kg}$ GUP gavage and receiving intraperitoneal administration of Escherichia coli O78). All birds were housed in a temperature- and light-controlled room with artificial light and ad libitum access to mash feed and fresh water during the entire experimental period. Meanwhile, the brooding temperature was maintained at 32 to $35^{\circ} \mathrm{C}$ for 7 days and then gradually decreased by $1{ }^{\circ} \mathrm{C}$ every 2 days until a final temperature of $22^{\circ} \mathrm{C}$ was reached at day 21 . In addition, all birds were vaccinated according to a routine immunization program. All birds were inoculated with an inactivated infectious bursal disease vaccine on days 7 and 21 and with an infectious bursa disease vaccine on day 14 . Antimicrobial and anticoccidial drugs were not added to the basal diet, which was formulated per the National Research Council to meet the nutritional requirements of broilers.

On the 8th and 15th days after drug administration, 6 broilers were taken from each group. Blood was collected and placed at room temperature for $1 \mathrm{~h}$ and centrifuged at 3000 $\mathrm{r} / \mathrm{min}$ for $10 \mathrm{~min}$ in a freezing centrifuge; the supernatant was removed and stored in a refrigerator at $-20^{\circ} \mathrm{C}$ for testing. The small intestine was collected and stored in $4 \%$ neutral paraformaldehyde fixative for paraffin section production. The proximal end of the cecum was tied tightly with rope; the cecum was cut at the ligation site and placed into a sterilized prelabeled $5 \mathrm{~mL} \mathrm{EP}$, which was immediately placed into liquid nitrogen (or quickly placed into the $-20^{\circ} \mathrm{C}$ refrigerator and immediately stored in the $-80^{\circ} \mathrm{C}$ refrigerator), and sent to the testing company for $16 \mathrm{~S}$ rDNA sequencing and analysis of intestinal flora changes. The small intestine segment was placed in RNA preservation solution (Beijing Baitech Biotechnology Co., LTD.) and stored at $-80^{\circ} \mathrm{C}$ to determine the mRNA expression of intestinal tight junction proteins (ZO-1, claudin-1, occludin).

ELISA assay: The levels of plasma endotoxin, diamine oxidase (DAO) and D-lactic acid (DLA) were measured using test kits (Shanghai Lanji Biotechnology Co., LTD) according to the manufacturer's instructions.

16S rDNA high throughput sequencing: Microbial diversity was based on the Illumina HiSeq sequencing platform, and the paired end sequencing method was used to construct a small fragment library for sequencing. Read assembly, filtering, OTU (operational taxonomic unit) clustering, and species annotation and abundance analysis can reveal the species composition of samples. Further alpha diversity, beta diversity and significant 
species diversity analyses were carried out to explore the differences between samples.

The fecal microbiomes for 60 fecal samples collected from six animals in the control group, model group, high-dose GUP group, medialdose GUP group and low-dose GUP group were examined using 16S rDNA gene sequencing by Beijing Baimaike Biotechnology Co. LTD. Total genomic DNA was extracted with a QIAamp DNA Stool Mini Kit (QIAGEN, Germany) according to the manufacturer's instructions. The DNA concentration and purity were tested by a Qubit 2.0 DNA test kit (Life Technologies, USA) and $1 \%$ agarose gel electrophoresis. DNA was stored at $-20^{\circ} \mathrm{C}$ until further processing. The general primers $338 \mathrm{~F}$ (5'- ACTCCTACGGGAGGCAGCA-3') and 806R (5'- GGACTACHVGGGTWTCTAAT-3') were used to amplify the V3-V4 hypervariable regions of the bacterial 16S rDNA for the Illumina MiSeq sequencing platform. Each PCR product was quantified and gradually diluted to obtain a stock solution at $1 \mu \mathrm{g} / \mathrm{mL}$.

PCR amplification products were purified, quantified and homogenized to form a sequencing library. The established library was first tested, and the qualified library was sequenced by Illumina HiSeq 2500. The original image data files obtained by highthroughput sequencing (such as Illumina HiSeq and other sequencing platforms) were transformed with sequenced reads by base calling analysis, and the results were stored in FASTQ (referred to as fq) file format, which contained sequence information of sequencing sequences (reads) and their corresponding sequencing quality information.

Data preprocessing: According to the overlap relation between PE reads, the double-end sequence data obtained from HiSeq sequencing were merged into one sequence tag, and quality control and filtration of reads were conducted.The three main steps were as follows:

1) PE reads assembly: FLASH v1.2.7 software was used to overlap the reads of each sample, and the assembled sequence obtained was called Raw Tags data.

2) Tag filtering: Trimmomatic v0.33 software was used to filter the assembled Raw Tags and obtain Clean Tags.

3) Chimera removal: UCHIME v4.2 software was used to identify and remove chimera sequences and obtain the final effective tags.

Information analysis content: OTU, diversity and difference analyses were conducted (see analysis results for details).

Quantitative Real-time PCR: Total RNA was extracted from chicken intestine tissues. The chicken intestinal tissue samples were ground with liquid nitrogen and extracted with a TransZol Up Plus RNA Kit. RNA concentration was determined by Nanodrop; $1 \mu \mathrm{L}$ RNA was taken, diluted 5 times, and analyzed by electrophoresis with a $1.5 \%$ agarose gel. Next, cDNA was generated from each total RNA sample with a TransScript II All-in-One FirstStrand cDNA Synthesis SuperMix for qPCR (One-Step gDNA Removal) PrimeScript RT reagent kit (TaKaRa, China). The reverse transcription reaction system and conditions were as follows Table 1. The primer sequences used are listed in Table 2 and qPCR reaction system and conditions of cDNA on Table 3 . The levels of tight junction protein mRNA were detected with a Bio-Rad CFX96 Real-Time System (Bio-Rad, USA).

Statistical Analysis: Data are expressed as the mean \pm standard deviation. Data were analyzed using SPSS v 17.0. Statistical significance was determined by one-way analysis of variance (ANOVA). Differences between two groups were analyzed by Tukey's test. Statistical significance was set at $P<0.05$.

\section{RESULTS}

Effect of Glycyrrhiza uralensis polysaccharide on intestinal mucosal barrier function in chickens: The indicators of intestinal mucosal barrier function in the plasma of chickens were consequently measured. ELISA 
Table 1. Reverse transcription reaction system of RNA ( $20 \mathrm{uL})$

\begin{tabular}{lcl}
\hline Reagent & System & \multicolumn{1}{c}{ Conditions } \\
\hline Total RNA & $1 \mu \mathrm{g}$ & Reverse: $55^{\circ} \mathrm{C} 30 \mathrm{~min}$ \\
5×TS II All-in-One SuperMix for qPCR & $4 \mu \mathrm{L}$ & Reverse: $85^{\circ} \mathrm{C} 5 \mathrm{~s}$ \\
gDNA Remover & $1 \mu \mathrm{L}$ & After the reaction, add $80 \mu \mathrm{L}$ RNase-free \\
RNase-free Water & Up to $20 \mu \mathrm{L}$ & water to dilute the cDNA \\
\hline
\end{tabular}

Table 2. Primers used for quantitative RT-PCR of mucosal barrier function

\begin{tabular}{|c|c|c|c|c|}
\hline $\begin{array}{l}\text { Target } \\
\text { gene }\end{array}$ & $\begin{array}{c}\text { Accession } \\
\text { number }\end{array}$ & Primer sequence 52'-32' & $\begin{array}{c}\text { Annealing } \\
\text { temperature } /{ }^{\circ} \mathrm{C}\end{array}$ & $\begin{array}{l}\text { Products } \\
\text { length } / \mathrm{bp}\end{array}$ \\
\hline$\overline{\beta \text {-Actin }}$ & NM_205518.1 & $\frac{\text { F:ACCGCAAATGCTTCTAAACC }}{\text { R:ATAAAGCCATGCCAATCTCG }}$ & 59 & 104 \\
\hline $\mathrm{ZO}-1$ & XM_015278981.2 & $\begin{array}{l}\text { F:CAACGTAGTTCTGGCATTATTCG } \\
\text { R:GGGCACAGCCTCATTCTCAT }\end{array}$ & 60 & 188 \\
\hline Claudin-1 & NM_001013611.2 & $\frac{\text { F:TGGAGGATGACCAGGTGAAG }}{\text { F:TGGAGGATGACCAGGTGAAG }}$ & 60 & 137 \\
\hline Occludin & NM_205128.1 & $\begin{array}{l}\text { F:AGACGCGCAGTAAGATCTGG } \\
\text { R:GGGCTTCTCCGAGTAGGCAA }\end{array}$ & 60 & 161 \\
\hline
\end{tabular}

Table 3. qPCR reaction system and conditions of cDNA

\begin{tabular}{lcl}
\hline \multicolumn{1}{c}{ Reagent } & System & \multicolumn{1}{c}{ Conditions of qPCR } \\
\hline Forward Primer $(10 \mu \mathrm{M})$ & $0.4 \mu \mathrm{L}$ & Predenaturation at $94^{\circ} \mathrm{C}$ for $30 \mathrm{~s}$ \\
Reverse Primer $(10 \mu \mathrm{M})$ & $0.4 \mu \mathrm{L}$ & Denaturation at $94^{\circ} \mathrm{C}$ for $5 \mathrm{~s}$ \\
$2 \times$ TransStart Top Green qPCR SuperMix & $10 \mu \mathrm{L}$ & Annealing at $60^{\circ} \mathrm{C}$ for $30 \mathrm{~s}$ \\
Template(cDNA) & $2 \mu \mathrm{L}$ & $72^{\circ} \mathrm{C}$ extended $10 \mathrm{~s}$ \\
ddH2O to final volume & $20 \mu \mathrm{L}$ & 40 cycles Draw Dissociation Stage \\
\hline
\end{tabular}

data showed that the levels of plasma endotoxin, DAO and D-LA in chickens of the model group were significantly higher than those in chickens of the control group on the 8th day and the 15th day after administration $(\mathrm{P}<0.05)$. The levels of endotoxin, DAO and D-LA in the different GUP group were all significantly reduced compared with the model group on the 8th day and the 15th day after administration $(\mathrm{P}<0.05)$. As the dose of GUP increased from 5 to $10 \mathrm{mg} / \mathrm{kg}$ the content of endotoxin, DAO and D-LA decreased, especially when the supplemental dose of GUP was $10 \mathrm{mg} / \mathrm{kg} \mathrm{bw} \cdot \mathrm{d}$ (medial-dose GUP group).
The content of endotoxin, DAO and D-LA was close to the control group on the 8th day and the 15th day after administration. These results indicated that GUP could facilitate injury to intestinal barrier function (Table 4).

16S rDNA high throughput sequencing analysis: The analysis of the molecular ecology of fecal flora was performed by $16 \mathrm{~S}$ rDNA high throughput sequencing. Six samples from different groups were examined.

OTU clustering and annotation: The trimmed and assembled sequences were clustered at $97 \%$ 
Table 4. Effects of GUP on serum diamine oxidase activity, D-LA concentration and endotoxin content of broilers challenged with $E$. coli 078

\begin{tabular}{|c|c|c|c|c|c|c|c|c|}
\hline \multirow[t]{2}{*}{ Group project } & \multicolumn{2}{|c|}{ Endotoxin } & DA & & D-LA & Endotox & DAO & D-LA \\
\hline & \multicolumn{5}{|c|}{ On the 8th day after administration } & \multicolumn{3}{|c|}{ On the 15th day after administration } \\
\hline Model group & $70.36 \pm 7.30^{\mathrm{a}}$ & $20.97 \pm$ & $00^{\mathrm{a}}$ & 56. & $0 \pm 5.91^{\mathrm{a}}$ & $73.87 \pm 6.63^{\mathrm{a}}$ & $21.62 \pm 3.32^{\mathrm{a}}$ & $\overline{58.75 \pm 6.49^{\mathrm{a}}}$ \\
\hline Control group & $43.28 \pm 8.74^{\mathrm{d}}$ & $15.58 \pm$ & $37^{\mathrm{bc}}$ & 38. & $9 \pm 3.49^{c}$ & $42.35 \pm 9.03^{\mathrm{cd}}$ & $15.06 \pm 2.28^{\mathrm{bc}}$ & $38.95 \pm 2.39^{\circ}$ \\
\hline $\begin{array}{l}\text { Low-dose } \\
\text { GUP group }\end{array}$ & $61.16 \pm 6.16^{\mathrm{b}}$ & $17.89 \pm$ & $96 b$ & 48. & $9 \pm 4.82^{b}$ & $59.25 \pm 6.26^{\mathrm{b}}$ & $16.84 \pm 2.27^{\mathrm{b}}$ & $45.92 \pm 3.06^{\mathrm{b}}$ \\
\hline $\begin{array}{l}\text { Medial-dose } \\
\text { GUP group }\end{array}$ & $52.74 \pm 5.49^{\mathrm{c}}$ & $16.09 \pm$ & $30^{\mathrm{b}}$ & 39 & $8 \pm 5.60^{c}$ & $49.82 \pm 7.17^{\mathrm{c}}$ & $16.00 \pm 2.44^{\mathrm{b}}$ & $37.04 \pm 6.62^{\mathrm{c}}$ \\
\hline $\begin{array}{l}\text { High-dose } \\
\text { GUP group }\end{array}$ & $42.97 \pm 2.35^{\mathrm{d}}$ & $14.50 \pm$ & & 35 & $0 \pm 2.55^{\mathrm{c}}$ & $35.34 \mathrm{~d} \pm 2.05^{\mathrm{d}}$ & $12.12 \pm 3.00^{\mathrm{c}}$ & $32.49 \pm 3.38^{\mathrm{c}}$ \\
\hline
\end{tabular}

The lower case of the same word on the shoulder of the same column means that the difference is not significant $(P>0.05)$, and the different letters indicate that the difference is significant $(P<0.05)$

similarity by calling uclust from QIIME. According to the obtained abundance matrix of OTUs, the number of OTUs in each group was calculated using $\mathrm{R}$ software, and the proportions for shared and unique OTUs were intuitively shown in a Venn diagram. The total OTUs obtained in each group were as follows: There were 513 shared OTUs among the five groups on the $8^{\text {th }}$ day after administration and 516 shared OTUs among the five groups on the $15^{\text {th }}$ day after administration. The OTUs of the low-dose GUP, medial-dose GUP, highdose GUP, control and model groups were 545, 551, 542, 531 and 533, respectively, and the unique OTUs of the low-dose GUP, medialdose GUP, high-dose GUP, control and model groups were $3,5,4,1$ and 0 , respectively, on the $8^{\text {th }}$ day after administration (Fig. 2a). The OTUs of the low-dose GUP, medial-dose GUP, high-dose GUP, control and model groups were $540,547,543,540$ and 547, respectively, and the unique OTUs of the low-dose GUP, medialdose GUP, high-dose GUP, control and model groups were $3,3,4,3,3$, respectively, on the $15^{\text {th }}$ day after administration (Fig.1b).

Alpha diversity reflects the individual sample species abundance, richness and species diversity; there are numerous indexes: Chao1, Ace, Shannon, and Simpson. Chao1 and Ace indexes measure the species abundance of the number of species. Shannon and Simpson indexes are used to measure species diversity and are affected by species abundance and community evenness in sample communities. In the case of the same species abundance, the greater the evenness of each species in the community, the greater the diversity of the community, the greater the Shannon index value, and the smaller the Simpson index value, indicating a higher species diversity of the sample.

As shown in Fig. 2a, Fig. 2b, the species richness (Chao, ACE) was higher in the caeca of broilers fed GUP than in those of the model group, which indicated that more bacterial species were detected in the GUP group on the 8th day after administration. The community diversity (Shannon, Simpson) was higher in the GUP group on the 15th day after administration (Fig. 3c, Fig. 3d).

Beta diversity analysis is shown in Fig. 5a and Fig. 5b. It can compare the differences in species diversity. The nonmetric multidimensional calibration method (metricmetric, metricbar and dimensional scalingnmds) is a kind of sorting method suitable for ecological research and is used mainly to simplify the study object (sample or variable) of multidimensional space to the low 
dimensional space for positioning, analysis and classification while also retaining the data analysis method of the original relationship between objects. Similar to PCA or PCoA the distribution of the sample can be seen in the group intergroup.

The results of the NMDS analysis are shown in Fig. 5, different colors represent different groups, and the distance between points indicates the difference. When the stress is less than 0.2, the NMDS analysis shows that the NMDS analysis has certain reliability, and the closer the distance is, the higher the similarity. The results of NMDS (stress $=0.1377$ ) showed that the distribution of microorganisms in the five groups on the 8th day was different, and the difference was most significant in the medium-dose group compared with the other groups (Fig. 4a). The NMDS (stress $=0.1314$ ) results showed similar microorganism distributions in the five groups on the 15th day (Fig. 4b).The distribution was evidently different, although a few samples overlapped, in the five groups on the 8th day compared with the five groups on the 15th day. Thus, we may conclude that GUP could promote intestinal microbial diversity on the eighth day after administration, and the effect was best in the medium dose group.

Phylum-level microbiota analysis showed that Bacteroidetes and Firmicutes were the two most dominant phyla (Fig. 5). Cluster analysis revealed that Bacteroidetes and Firmicutes comprised the main phyla in each group, but the composition was different. The abundances of Bacteroidetes in the high-dose GUP, medialdose GUP and low-dose GUP treatment groups were $7.73 \%, 35.78 \%$ and $11.74 \%$, respectively, and significantly higher than those in the control and model groups $(5.03 \%$ and $0.07 \%$, respectively) on the $8^{\text {th }}$ day after administration. The abundance of Faecalibacterium in the model group was $17.63 \%$, which was higher than that in the control group (15.63\%). The abundance of Faecalibacterium in the high-dose GUP, medial-dose GUP and low-dose GUP groups was $17.63 \%, 5.23 \%$ and $13.84 \%$, respectively, and the medial-dose and low-dose groups were significantly lower than the model group $(17.12 \%)$. The abundance ratios of Bacteroidetes/Firmicutes in the high-dose GUP, medial-dose GUP and low-dose GUP groups were $0.44,6.84,0.85$, respectively, which were higher than those in the control and model groups $(0.32$ and 0.0041 , respectively, $\mathrm{P}<0.05)$ on the $8^{\text {th }}$ day after administration (Fig. 5a).

The abundances of Bacteroidetes in the high-dose GUP, medial-dose GUP and lowdose GUP groups were $41.73 \%, 39.48 \%$ and $31.79 \%$, respectively, which were higher than that in the model group $(26.08 \%)$ on the $15^{\text {th }}$ day after administration. The abundances of Faecalibacterium in the high-dose GUP, medialdose GUP and low-dose GUP groups were $10.70 \%, 9.18 \%$ and $12.42 \%$, respectively, and low compared with the those of the control and model groups $(12.39 \%$ and $13.93 \%$, respectively). The abundance ratios of Bacteroidetes / Firmicutes in the high-dose GUP, medial-dose GUP and low-dose GUP groups were $3.9,4.30$ and 2.56, respectively, which were higher than those in the control and model groups (3.51 and 1.87, respectively, $\mathrm{P}<0.05$ ) on the $15^{\text {th }}$ day after administration (Fig. 5b). Analysis of the composition of bacteria at the phylum level is shown in Fig. 7. Cluster analysis revealed that Bacteroidetes and Firmicutes comprised the main phyla in each group, but the composition was different (Fig. 6). The abundances of Bacteroidetes in the high-dose GUP, medial-dose GUP and low-dose GUP groups were $7.74 \%, 35.80 \%$ and $11.76 \%$, respectively, which was higher than those in the control and model groups $(5.03 \%$ and $0.07 \%$, respectively) on the 8 th day after administration. The abundance of Faecalibacterium in the model group was $95.93 \%$, which was higher than that in the control group $(90.06 \%)$. The abundance ratios of the Bacteroidetes/Firmicutes in the high-dose GUP, medial-dose GUP and low-dose GUP groups were $0.09,0.57$ and 0.14 , respectively, which were higher than those in the control and model groups (0.06 and 0.00073 , respectively, 
Indian Journal of Animal Health, December, 2021

\section{Effects of refined Glycyrrhiza uralensis on gut health of broilers}
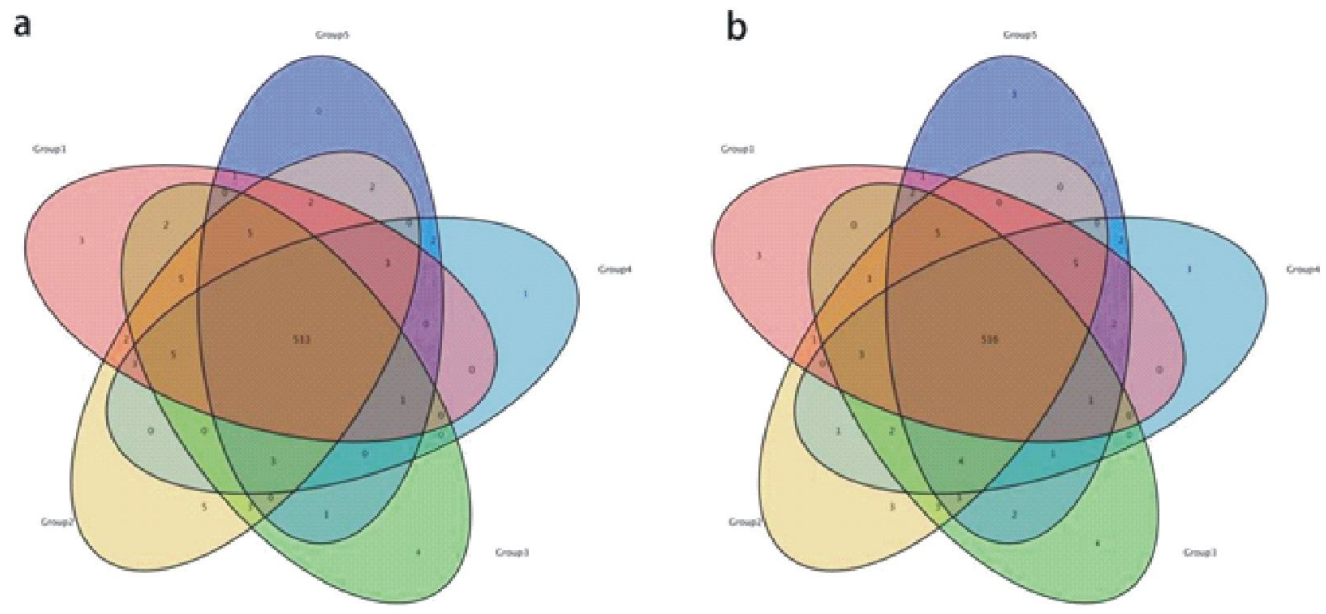

Note: Venn diagram of operational taxonomic unit (OTU) distribution. Each ellipse represents one of the samples. The overlapping regions between the ellipses represent shared OTUs between samples. The value for each region represents the number of OTUs corresponding to the region. a) 8th day after administration; b) 15th day after administration; group 1: low-dose GUP; group 2: medial-dose GUP; group 3: high-dose GUP; group 4: control group 5: model.

Fig. 1. Venn diagram
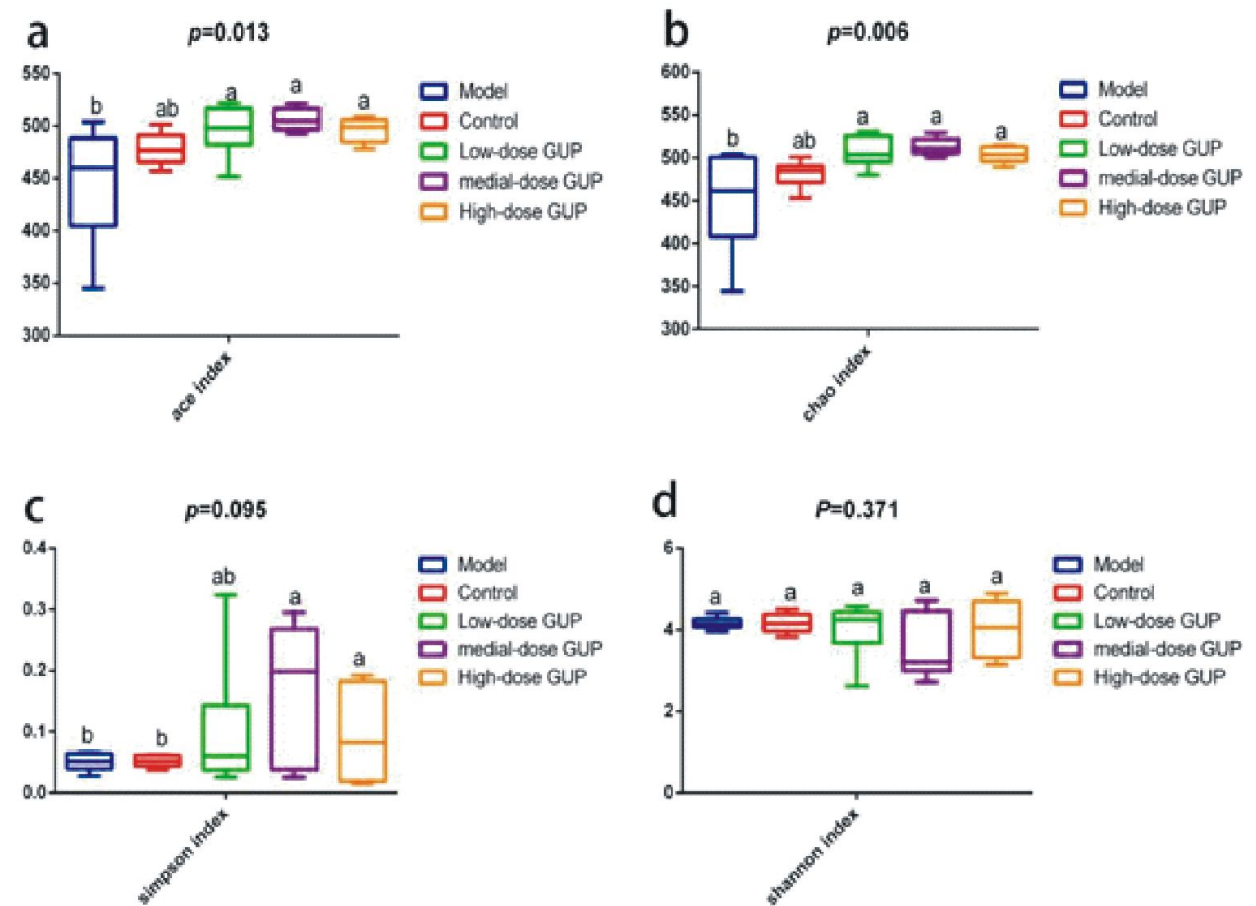

Note: a,b,c,d: Means Alpha diversity of five groups on the 8th day after administration All data are presented as the mean \pm SD $(n=6)$

Fig. 2. Alpha diversity 
Indian Journal of Animal Health, December, 2021

Effects of refined Glycyrrhiza uralensis on gut health of broilers
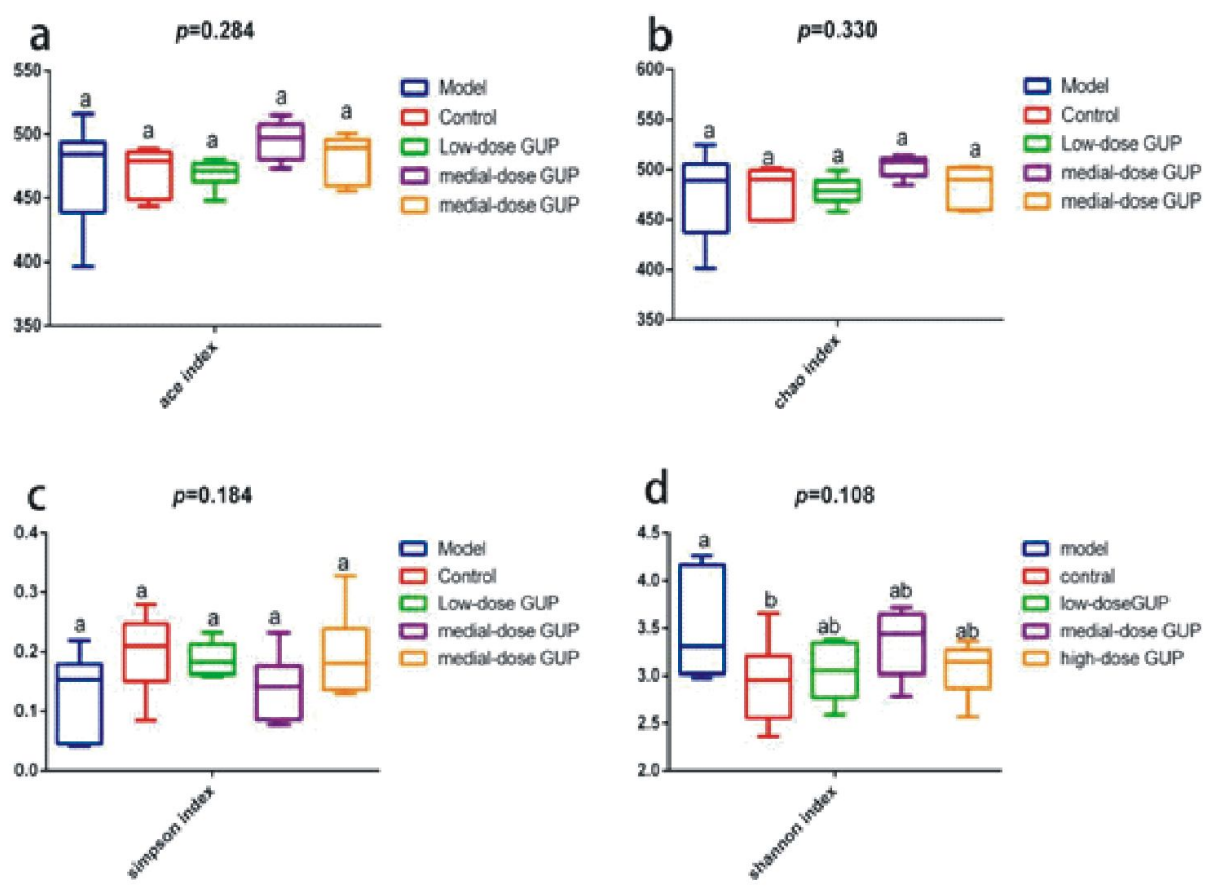

Note: a,b,c,d: Means Alpha diversity of five groups on the 15th day after administration All data are presented as the mean $\pm \operatorname{SD}(n=6)$

Fig. 3. Alpha diversity
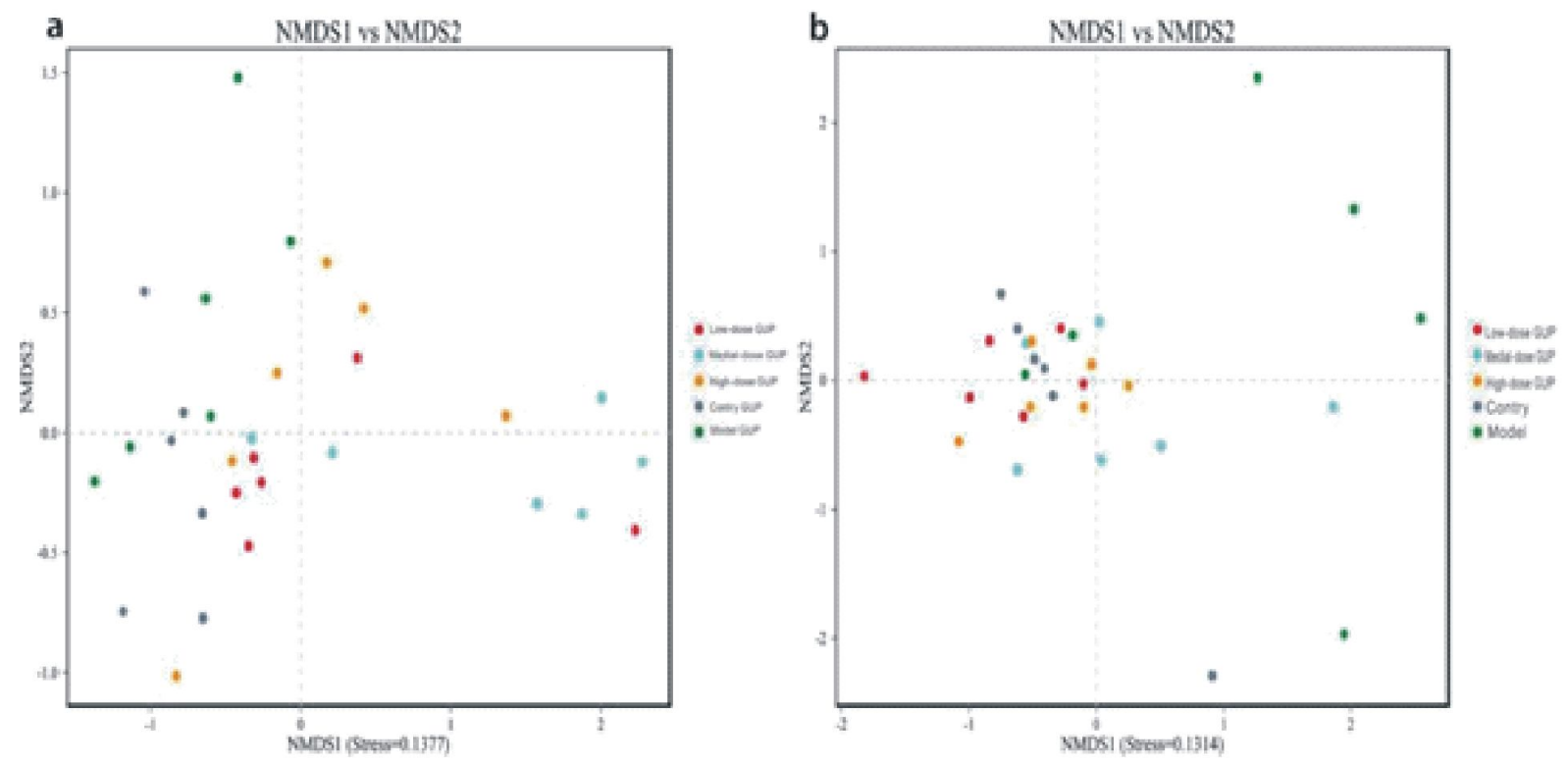

Fig. 4. NMDS1 analysis 
Indian Journal of Animal Health, December, 2021

\section{Effects of refined Glycyrrhiza uralensis on gut health of broilers}
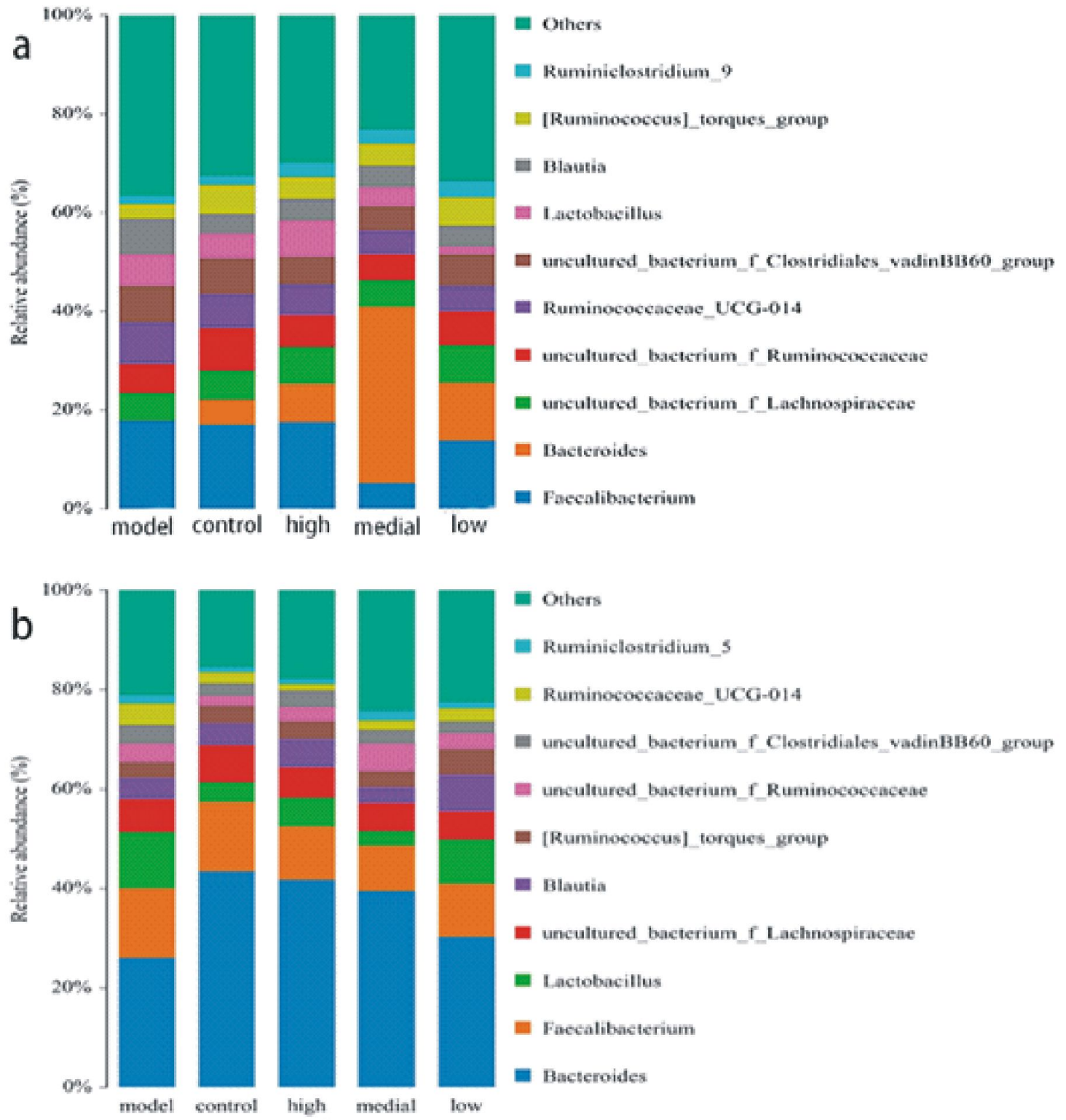

Fig. 5. Species composition and relative abundance of cecum flora at major genus level in different groups 
Indian Journal of Animal Health, December, 2021

Effects of refined Glycyrrhiza uralensis on gut health of broilers
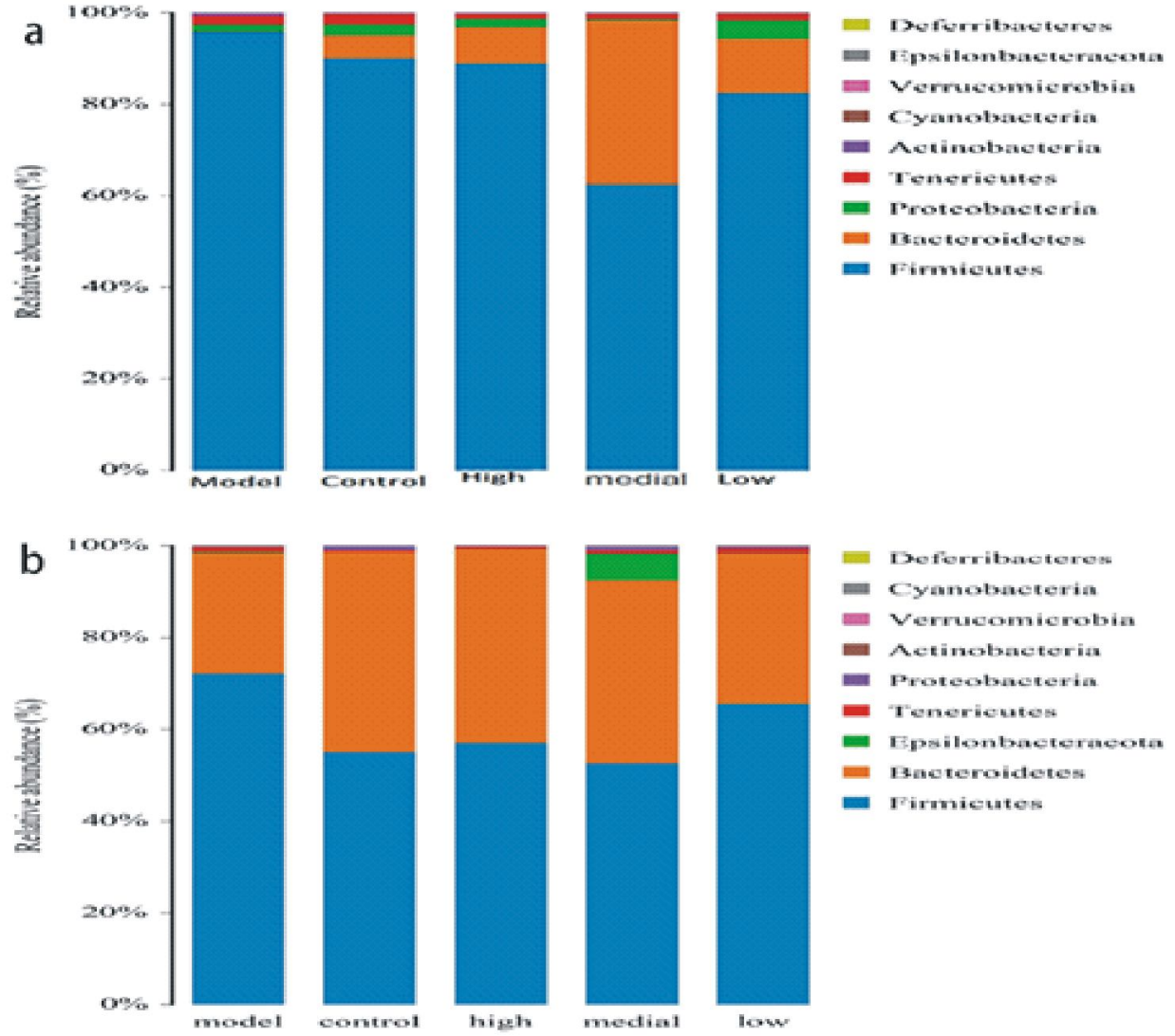

Fig. 6. Species composition and relative abundance of cecum flora at major phyla level in
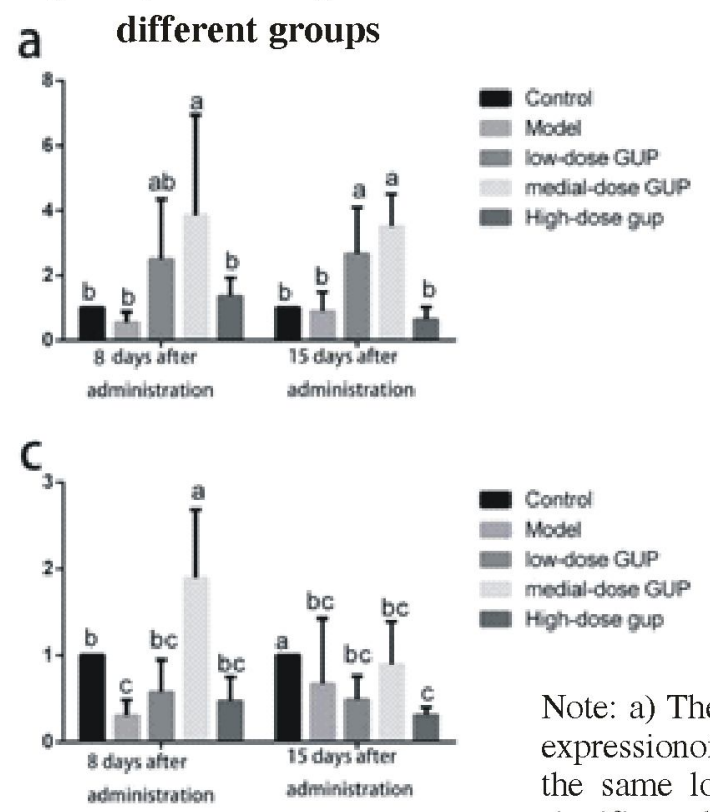

$$
\begin{aligned}
& \text { Control } \\
& \text { Model } \\
& \text { lowdose GUP } \\
& \text { modial-dose GUP } \\
& \text { High-dose gup }
\end{aligned}
$$

b

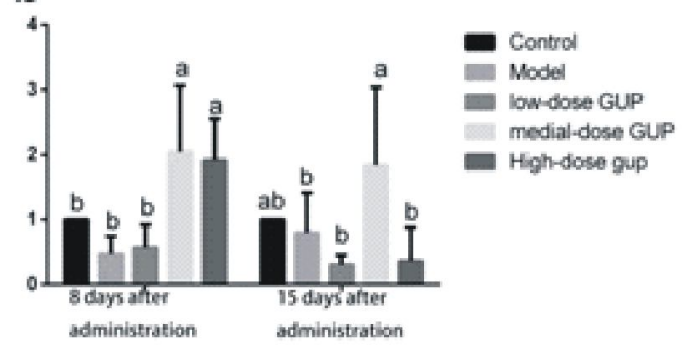

Note: a) The expression of ZO-1; b) the expression of occludin; c): the expressionof claudin-1. All data are presented as the mean $\pm \mathrm{SD}(\mathrm{n}=5)$, the same lowercase letters at the top of the bar chart indicated no significant difference $(P>0.05)$, while different lowercase letters indicated significant difference $(P<0.05)$.

Fig. 7. Effects of Glycyrrhiza uralensis polysaccharide on the relative expression of mRNA of small intestine compact junction protein 
$P<0.05$ ) on the 8th day after administration (Fig. $6 a)$.

The abundances of Bacteroidetes in the high-dose GUP, medial-dose GUP and lowdose GUP groups were $42.43 \%, 39.89 \%$ and $33.08 \%$, respectively, which were higher than that in the model group $(26.23 \%)$ on the $15^{\text {th }}$ day after administration. The abundance of Faecalibacterium in the model group was $72.18 \%$, which was higher than that in the control group (55.12\%). The abundance ratios of Bacteroidetes/Firmicutes in the high-dose GUP, medial-dose GUP and low-dose GUP groups were $0.74,0.76$ and 0.50 , respectively, which were higher than that in the model group $(0.36, P<0.05)$ on the $15^{\text {th }}$ day after administration (Fig. 6b).

Effect of Glycyrrhiza uralensis polysaccharide on the expression of tight junction proteins: To further evaluate the intestinal mucosal barrier function, the expression of the tight junction proteins in the jejunal tissue of chickens was determined by qPCR. The data (Fig. 7) showed that the levels of ZO-1, occludin, and claudin- 1 in chickens of the model group were significantly lower than those in chickens of the control group on the 8th day and the 15th day after administration $(\mathrm{P}<0.05)$. The levels of ZO-1, occludin and claudin- 1 in chickens of different GUP groups were all increased compared with those of the model group on the 8th day after administration. On the 8th day after administration the mediumdose group showed significant differences in the expression of ZO-1, occludin and claudin1 and the high-dose group also showed significant differences in the expression of occludin (Fig. 7b). On the 15th day after administration, the ZO- 1 expression in chickens of the low-dose GUP and medial-dose GUP groups was significantly increased (Fig. 7a). The expression of occludin in chickens of the medial-dose GUP group was significantly increased (Fig. 7b).

\section{DISCUSSION}

Schedule 1. Relative abundance of intestinal flora in each group at the genus level

\begin{tabular}{cccccc}
\hline GenusGroup & $\begin{array}{c}\text { Model } \\
\text { group }\end{array}$ & $\begin{array}{c}\text { Control } \\
\text { group }\end{array}$ & $\begin{array}{c}\text { High- } \\
\text { dose GUP } \\
\text { group }\end{array}$ & $\begin{array}{c}\text { Medial- } \\
\text { dose GUP } \\
\text { group }\end{array}$ & $\begin{array}{c}\text { Low- } \\
\text { dose GUP } \\
\text { group }\end{array}$ \\
\hline
\end{tabular}

On the 8th day after administration

\begin{tabular}{lllllr}
\hline Bacteroides & $0.07 \%$ & $5.03 \%$ & $7.73 \%$ & $35.78 \%$ & $11.74 \%$ \\
\hline Faecalibacterium & $17.12 \%$ & $15.63 \%$ & $17.63 \%$ & $5.23 \%$ & $13.84 \%$ \\
\hline uncultured_bacterium_f_Lachnospiraceae & $7.68 \%$ & $5.89 \%$ & $7.52 \%$ & $5.38 \%$ & $7.57 \%$ \\
\hline uncultured_bacterium_f_Ruminococcaceae & $5.90 \%$ & $8.48 \%$ & $6.33 \%$ & $5.13 \%$ & $6.81 \%$ \\
\hline Ruminococcaceae_UCG-014 & $8.56 \%$ & $7.01 \%$ & $6.33 \%$ & $4.98 \%$ & $5.25 \%$ \\
\hline $\begin{array}{l}\text { uncultured_bacterium_f_Clostridiales_ } \\
\text { vadinBB60_group }\end{array}$ & $7.27 \%$ & $7.19 \%$ & $5.62 \%$ & $4.76 \%$, & $6.40 \%$ \\
\hline Lactobacillus & & & & \\
\hline Blautia & $6.52 \%$ & $5.14 \%$ & $7.27 \%$ & $3.94 \%$ & $1.55 \%$ \\
\hline [Ruminococcus]_torques_group & $7.14 \%$ & $3.97 \%$ & $4.47 \%$ & $4.32 \%$ & $4.23 \%$ \\
\hline Ruminiclostridium_9 & $3.02 \%$ & $5.77 \%$ & $4.42 \%$ & $4.53 \%$ & $5.84 \%$ \\
\hline Others & $1.68 \%$ & $1.89 \%$ & $2.85 \%$ & $2.84 \%$ & $3.19 \%$ \\
\hline On the 15th day after administration & $36.53 \%$ & $32.50 \%$ & $29.84 \%$ & $23.12 \%$ & $33.57 \%$ \\
\hline Bacteroides & $26.08 \%$ & $43.49 \%$ & \multicolumn{3}{c}{$41.73 \%$} \\
\hline
\end{tabular}


Schedule 1., Cont. ...

\begin{tabular}{lccccc}
\hline \multicolumn{1}{c}{ GenusGroup } & $\begin{array}{c}\text { Model } \\
\text { group }\end{array}$ & $\begin{array}{c}\text { Control } \\
\text { group }\end{array}$ & $\begin{array}{c}\text { High- } \\
\text { dose GUP } \\
\text { group }\end{array}$ & $\begin{array}{c}\text { Medial- } \\
\text { dose GUP } \\
\text { group }\end{array}$ & $\begin{array}{c}\text { Low- } \\
\text { dose GUP } \\
\text { group }\end{array}$ \\
\hline Faecalibacterium & $13.93 \%$ & $12.39 \%$ & $10.70 \%$ & $9.18 \%$ & $12.42 \%$ \\
\hline Lactobacillus & $12.94 \%$ & $3.98 \%$ & $5.82 \%$ & $2.95 \%$ & $7.81 \%$ \\
\hline uncultured_bacterium_f_Lachnospiraceae & $6.53 \%$ & $7.42 \%$ & $6.14 \%$ & $5.64 \%$ & $5.55 \%$ \\
\hline Blautia & $4.44 \%$ & $4.53 \%$ & $5.84 \%$ & $3.16 \%$ & $7.79 \%$ \\
\hline Ruminococcus]_torques_group & $3.06 \%$ & $3.49 \%$ & $3.46 \%$ & $3.23 \%$ & $4.73 \%$ \\
\hline uncultured_bacterium_f_Ruminococcaceae & $3.76 \%$ & $1.92 \%$ & $2.84 \%$ & $5.54 \%$ & $3.12 \%$ \\
\hline uncultured_bacterium_f_Clostridiales_- & $3.76 \%$ & $2.47 \%$ & $3.35 \%$ & $2.69 \%$ & $2.70 \%$ \\
vadinBB60_group & & & & & \\
\hline Ruminococcaceae_UCG-014 & $4.36 \%$ & $2.36 \%$ & $1.32 \%$ & $1.97 \%$ & $2.33 \%$ \\
\hline Ruminiclostridium_5 & $1.64 \%$ & $0.95 \%$ & $1.03 \%$ & $2.00 \%$ & $1.30 \%$ \\
\hline Others & $21.06 \%$ & $15.46 \%$ & $17.76 \%$ & $24.14 \%$ & $20.45 \%$ \\
\hline
\end{tabular}

Schedule 2. Relative abundance of intestinal flora in each group of samples at the phylum level

\begin{tabular}{llcccc}
\hline \multicolumn{1}{c}{ Phylum Group } & $\begin{array}{c}\text { Model } \\
\text { group }\end{array}$ & $\begin{array}{c}\text { Control } \\
\text { group }\end{array}$ & $\begin{array}{c}\text { High- } \\
\text { dose GUP } \\
\text { group }\end{array}$ & $\begin{array}{c}\text { Medial- } \\
\text { dose GUP } \\
\text { group }\end{array}$ & $\begin{array}{c}\text { Low- } \\
\text { dose GUP } \\
\text { group }\end{array}$ \\
\hline On the 8th day after administration & & & & & \\
\hline Firmicutes & $95.93 \%$ & $90.06 \%$ & $89.05 \%$ & $62.58 \%$ & $82.49 \%$ \\
\hline Bacteroidetes & $0.07 \%$ & $5.03 \%$ & $7.74 \%$ & $35.80 \%$ & $11.76 \%$ \\
\hline Proteobacteria & $1.37 \%$ & $2.32 \%$ & $1.89 \%$ & $0.22 \%$ & $4.17 \%$ \\
\hline Tenericutes & $2.02 \%$ & $2.34 \%$ & $1.08 \%$ & $1.13 \%$ & $1.36 \%$ \\
\hline Actinobacteria & $0.47 \%$ & $0.19 \%$ & $0.15 \%$ & $0.12 \%$ & $0.11 \%$ \\
\hline Cyanobacteria & $0.11 \%$ & $0.04 \%$ & $0.04 \%$ & $0.11 \%$ & $0.06 \%$ \\
\hline Verrucomicrobia & $0.02 \%$ & $0.01 \%$ & $0.04 \%$ & $0.04 \%$ & $0.04 \%$ \\
\hline Epsilonbacteraeota & $0.00 \%$ & $0.00 \%$ & $0.01 \%$ & $0.01 \%$ & $0.00 \%$ \\
\hline Deferribacteres & $0.00 \%$ & $0.00 \%$ & $0.00 \%$ & $0.00 \%$ & $0.00 \%$ \\
\hline On the 15th day after administration & & & & & \\
\hline Firmicutes & $72.18 \%$ & $55.12 \%$ & $57.03 \%$ & $52.65 \%$ & $65.54 \%$ \\
\hline Bacteroidetes & $26.23 \%$ & $43.56 \%$ & $42.43 \%$ & $39.89 \%$ & $33.08 \%$ \\
\hline Epsilonbacteraeota & $0.22 \%$ & $0.00 \%$ & $0.00 \%$ & $5.80 \%$ & $0.00 \%$ \\
\hline Tenericutes & $1.02 \%$ & $0.39 \%$ & $0.33 \%$ & $0.68 \%$ & $0.90 \%$ \\
\hline Proteobacteria & $0.17 \%$ & $0.78 \%$ & $0.03 \%$ & $0.75 \%$ & $0.26 \%$ \\
\hline Actinobacteria & $0.16 \%$ & $0.13 \%$ & $0.14 \%$ & $0.19 \%$ & $0.15 \%$ \\
\hline Verrucomicrobia & $0.03 \%$ & $0.02 \%$ & $0.02 \%$ & $0.03 \%$ & $0.04 \%$ \\
\hline Cyanobacteria & $0.01 \%$ & $0.00 \%$ & $0.01 \%$ & $0.00 \%$ & $0.03 \%$ \\
\hline Deferribacteres & $0.00 \%$ & $0.00 \%$ & $0.00 \%$ & $0.00 \%$ & $0.00 \%$ \\
\hline
\end{tabular}


There are tens of millions of microorganisms in the intestines of animals, collectively referred to as gut microbiota. The gastrointestinal tract is constantly exposed to our diet, and commensal bacteria and xenobiotics all have an effect on host physiology (Li et al., 2016). In health, the function of the gastrointestinal microbiome may be considered to be in dynamic equilibrium with the host, which plays both local and remote roles (Díaz Carrasco et al., 2018). However, "disequilibrium" may lead to various intestinal diseases. Risk factors for various intestinal diseases include not only changes in the microbiota but also incomplete intestinal barrier function and intestinal hypoxia/ischemia (Zhang et al., 2018).

This study investigated the effect of GUP on the intestinal flora structure and intestinal epithelial barrier function of broilers after $E$. coli O78 infection. The results of pathological section (Fig. 1) after dietary GUP supplementation revealed that the morphological structure of the small intestinal villus improved. The intestinal wall was complete, and the structure was clear. This result suggested that GUP could restore the damaged mucosa to some degree.

Many studies have shown that as the diversity of intestinal microorganisms in animals increases, and the incidence of intestinal diseases (Cukrowska et al., 2017), such as enteritis, severe acute pancreatitis, will be reduced. The results of $16 \mathrm{~S}$ rDNA highthroughput sequencing indicated that GUP increased the diversity of intestinal microbiota. The Venn diagram showed that on the 8th day after administration, the number of OTUs specific to each GUP group increased significantly more than that in the control group and the model group (the number of OTUs in the control group was more than that in the model group), while on the 15 th day after administration, there was no significant difference between the groups (Fig. 2). As shown in Fig. 3 and Fig. 4, the diversity of the total bacterial community increased during GUP treatment. At the 8th day, both Shannon and Simpson indexes indicated that the fecal microbiota diversity in E. coli O78-infected chickens fed GUP was significantly higher than that in the control group, but not higher than that in the GUP treatment $(\mathrm{P}<0.05)$. In a previous study (Cheng et al., 2018; He et al., 2019), an increased abundance of Firmicutes and a decreased abundance of Bacteroidetes were discovered in animals with intestinal diseases. These changes in gut microbial populations were also observed in our model group compared to the control group. Our data showed that GUP induced changes in microbial composition at the phylum level and genus level. The chickens in the model group showed a significant shift in the composition of intestinal flora, with a reduced percentage of Bacteroidetes and an increased proportion of Firmicutes. After GUP treatment, the abundance of Bacteroidetes increased, while the abundance of Firmicutes decreased, so the ratio of Bacteroidetes/Firmicutes increased significantly. It was reported that the declined abundance ratio of Bacteroidetes/Firmicutes is an indicator of microbial imbalance (Saxena et al., 2016; Xu et al., 2016; Oh et al., 2017 ); it has been suggested that GUP could improve dysbacteriosis in broilers with intestinal damage. GUP treatment decreased the abundance of the phylum Firmicutes.

In this study, the intestinal mucosal barrier function of chickens was tested. Gastrointestinal mucosa is an important barrier for close contact between numerous microorganisms, environmental antigens, the host immune system and digestive system (Zhu et al., 2018). Intestinal barrier defects have been associated with a broad range of diseases (Luo et al., 2019; Vargas-Robles et al., 2019). To maintain host homeostasis, intestinal microbial balance, intestinal epithelial integrity, and contact between intestinal microorganisms and epithelial cell surfaces must be limited and carefully controlled (Zmora et al., 2017). The barrier function of intestinal mucosa plays a key role in maintaining the balance between host and microbiota. TJs are the most important structures in the intestinal mucosal barrier 
(Kumar et al., 2018; Horioka et al., 2019). Occludin, claudins and junctional adhesion molecules are important components of tight junctions in the intestinal barrier and function as channel proteins. These transmembrane proteins are linked to the cytoskeleton by zonula occludens, which constitute TJs (Song et al., 2019). Studies have shown that intestinal barrier dysfunction is accompanied by down regulation of the expression of TJ proteins, including (ZO-1, occludin and claudin-1) mRNA (Awad et al., 2017). Diamine oxidase (DAO) is a highly active intracellular enzyme produced by intestinal epithelia, and it exists only in the intestinal mucosa and ciliated cells, whereas D-LA is the product of intestinal bacteria fermentation and endotoxin is the decomposition product of harmful bacteria (Awad et al., 2017; Horioka et al., 2019). The intact intestinal mucosa provides a barrier function to prevent DAO, D-LA and endotoxin from infiltrating the portal circulation, which may be used to quantitatively assess intestinal mucosal barrier function (Yang et al., 2019). So, in this study, mRNA expression of ZO-1, occludin and claudin-1 in the intestinal mucosa and serum DAO and D-LA activity and endotoxin content were used as indicators for intestinal permeability and mucosal damage.

In the present study, E. coli O78 challenge destroyed jejunal TJs, which was characterized by enhanced mRNA expression of ZO-1, occludin and claudin-1 in the intestinal mucosa medial-dose GUP group, and both the medialdose and high-dose GUP group showed increased occludin mRNA expression. These results show that GUP could repair intestinal barrier function by enhancing the expression of $\mathrm{TJ}$ proteins, including ZO-1, occludin and claudin-1. In this study, the serum DAO and

\section{REFERENCES}

Awad WA, Hess C and Hess M, 2017. Enteric pathogens and their toxin-induced disruption of the intestinal barrier through alteration of tight junctions in chickens. Toxins, 9(2): 60, doi: 10.3390/toxins9020060

Bian X, Wang TT, Xu M, Evivie SE, Luo GW et al.,
D-LA activity and endotoxin content of broilers infected by $E$. coli $\mathrm{O} 78$ were significantly increased. Compared with the model group, the serum endotoxin content was significantly decreased in the GUP treatment group and was negatively correlated with the drug dose on the 8th day and 15th day after administration. Serum DAO activity in the GUP treatment group decreased significantly compared with the model group, and with the increase in drug dose, the DAO activity decreased more on the 8th day and 15th day after oral administration of GUP. Serum D-LA activity in the GUP treatment group decreased significantly compared with that in the model group and was negatively correlated with the drug dose on the 8th day and 15th day after administration. This suggests that GUP can maintain intestinal barrier function by lowering serum DAO and D-LA activity and endotoxin content.

GUP can improve the morphology and structure of intestinal villi of intestinal damaged broilers, making the intestinal wall more complete and clearer. Feeding GUP resulted in a more diverse gut microbiome and changed the bacterial composition with an increased percentage of Bacteroidetes and a decreased proportion of Firmicutes. Meanwhile, oral GUP promoted intestinal barrier function by enhancing the mRNA expression of TJ proteins and reducing the serum DAO and D-LA activity and endotoxin content. Taken together, these findings indicate that GUP may be a therapy for the treatment or prevention of intestinal disease.

Conflict of interest: Authors have no conflict of interest in this study.

2016. Effect of lactobacillus strains on intestinal microflora and mucosa immunity in Escherichia coli $\mathrm{O} 157: \mathrm{H7}$ - induced diarrhea in mice. Curr Microbiol, 73(1): 65-70, doi: 10.1007/s00284016-1010-3

Cheng M, Zhang X, Zhu J, Cheng L, Cao J et al., 2018. 
A metagenomics approach to the intestinal microbiome structure and function in high fat diet-induced obesity mice fed with oolong tea polyphenols. Food Funct, 9(2): 1079-1087, doi: 10.1039/ c7fo01570d

Cukrowska B, Sowiñska A, Bierła JB, Czarnowska E, Rybak A et al., 2017. Intestinal epithelium, intraepithelial lymphocytes and the gut microbiota - Key players in the pathogenesis of celiac disease. World J Gastroenterol 23(42): 7505-7518, doi: 10.3748/wjg.v23.i42.7505

Díaz Carrasco JM, Redondo EA, Pin Viso ND, Redondo LM, Farber MD et al., 2018. Tannins and bacitracin differentially modulate gut microbiota of broiler chickens. Biomed Res Int: 1879168, doi: 10.1155/2018/1879168

He J, Hai L, Orgoldol K, Yi L, Ming L et al., 2019. High-throughput sequencing reveals the gut microbiome of the bactrian camel in different ages. Current Microbiol, 76(10), doi: 10.1007/s00284019-01689-6

Horioka K, Tanaka H, Isozaki S, Konishi H, Fujiya M et al., 2019. Acute colchicine poisoning causes endotoxemia via the destruction of intestinal barrier function: The curative effect of endotoxin prevention in a murine model. Dig Dis Sci, 65(1): 132-140, doi: 10.1007/s10620-019-05729-w

Hou YJ, jiang XW and Li ZJ, 2018. Repair of liver and intestinal mucosa damage caused by Escherichia coli. Chinese J Vet Med, 54(3): 47-50

Jian L, Li H, Chen J, He L, Du X et al., 2019. Dendrobium officinale polysaccharides alleviate colon tumorigenesis via restoring intestinal barrier function and enhancing anti-tumor immune response. Pharmacol Res: 104417, doi: 10.1016/ j.phrs.2019.104417

Kumar A, Chatterjee I, Anbazhagan AN, Jayawardena D, Priyamvada Set al., 2018. Cryptosporidium parvum disrupts intestinal epithelial barrier function via altering expression of key tight junction and adherens junction proteins. Cell Microbiol, 20(6): e12830, doi: 10.1111/cmi.12830

Li KJ, Chen W and Zhou X, 2017. Effects of dang shen polysaccharide on growth performance, immune function, blood routine and intestinal flora of black chicken. Sichuan Anim Husband Veterinary Med, 44(10): 32-34

Li Y, Xu Q, Huang Z, Lv L, Liu Get al., 2016. Effect of Bacillus subtilis CGMCC 1.1086 on the growth performance and intestinal microbiota of broilers. J Appl Microbiol, 120(1): 195-204, doi: 10.1111/ jam. 12972
Lian K-X, Zhu X-Q, Chen J, Liu J and Gu X-L, 2018. Selenylation modification: enhancement of the antioxidant activity of a Glycyrrhiza uralensis polysaccharide. Glycoconjugate J, 35: 243-253, doi: $10.1007 / \mathrm{s} 10719-018-9817-8$

Liao C-S, Wang X-L and Niu X-W 2015. Mediation of licorice polysaccharide on the disturbance of oxidization-antioxidant balance in macrophages induced by Salmonella typhimurium. Chinese J Vet Med, 35(06): 941-945

Lirong W, Dong Y and Hu J, 2011. Effects of licorice polysaccharide on antibody titer and body mass of new urban disease in young chickens. Acta Agriculturae Boreali Occidentalia Sinica, 20(6): 55-57

Luo J, Chen D, Mao X, He J, Yu B et al., 2019. Purified $\beta$-glucans of different molecular weights enhance growth performance of LPS-challenged piglets via improved gut barrier function and microbiota. Animals, 9(9): 602, doi: 10.3390/ani9090602

Oh JK, Pajarillo EAB, Chae JP, Kim IH, Yang DS et al., 2017. Effects of Bacillus subtilis CSL2 on the composition and functional diversity of the faecal microbiota of broiler chickens challenged with Salmonella gallinarum. J Animal Sci Biotechnol, 8: 1, doi: 10.1186/s40104-016-0130-8

Ping Y, Cui M, Li Y, Deng Y and Wu H, 2015. Effects of rhubarb on intestinal flora and toll-like receptors of intestinal mucosa in rats with severe acute pancreatitis. Pancreas, 44(5): 799-804, doi: 10.1097/MPA.0000000000000339

Qi Y-L, Li S-S, Qu D, Chen L-X, Gong R-Z et al, 2019. Effects of ginseng neutral polysaccharide on intestinal flora composition and diversity in mice. World J Tradit Chin Med, 44(4): 811-818, doi: 10.19540/j.cnki.cjcmm.20181129.002

Saxena S, Saxena VK, Tomar S, Sapcota D and Gonmei G, 2016. Characterisation of caecum and crop microbiota of Indian indigenous chicken targeting multiple hypervariable regions within 16S rRNA gene. Br Poult Sci, 57(3): 381-389, doi: 10.1080/00071668.2016.1161728

Song M, Ye J, Zhang F, Su H, Yang X et al., 2019. Chenodeoxycholic acid (CDCA) protects against the lipopolysaccharide-induced impairment of the intestinal epithelial barrier function via the FXRMLCK pathway. J Agric Food Chem, 67(32): 8868-8874, doi: 10.1021/acs.jafc.9b03173

Vargas-Robles H, Castro-Ochoa KF, Citalán-Madrid AF and Schnoor M, 2019. Beneficial effects of nutritional supplements on intestinal epithelial 
barrier functions in experimental colitis models in vivo. World J Gastroenterol, 25(30): 4181-4198, doi: 10.3748/wjg.v25.i30.4181

Wang R, Zhou G, Wang M, Peng Y and Li X, 2014. The Metabolism of polysaccharide from atractylodes macrocephala koidz and its effect on intestinal microflora. Evid Based Complement Alterna Med, 2014: 926381, doi: 10.1155/2014/ 926381

Wang W, Ren W, Li Z, Yue Y and Guo Y, 2017. Effects of yeast on immune and intestinal barrier function of broilers and its mechanism. Can J Anim Sci, 97: 136-144, doi: 10.1139/cjas-2015-0148

Wang X, Zhao L and Zhao 2015. Effects of Illumina high-throughput sequencing on intestinal flora of mice. Food science, 36(19): 222-226

Xiao S, Li Q, Hu K, He Y, Ai Q et al., 2018. Vitamin A and retinoic acid exhibit protective effects on necrotizing enterocolitis by regulating intestinal flora and enhancing the intestinal epithelial barrier. Arch Med Res, 49(1): 1-9, doi: 10.1016/ j.arcmed.2018.04.003

Xu Y, Yang H, Zhang L, Su Y, Shi D et al., 2016. Highthroughput sequencing technology to reveal the composition and function of cecal microbiota in Dagu chicken. BMC Microbiol, 16(1): 259, doi: 10.1186/s12866-016-0877-2

Xue M, Ji X, Liang H, Liu Y, Wang B et al., 2018. The effect of fucoidan on intestinal flora and intestinal barrier function in rats with breast cancer. Food Funct, 9(2): 1214-1223, doi: 10.1039/ c7fo01677h

Yang L, Liu G, Lian K, Quai Y, Zhang B et al., 2019. Dietary leonurine hydrochloride supplementation attenuates lipopolysaccharide challenge-induced intestinal inflammation and barrier dysfunction by inhibiting the NF-kB/MAPK signaling pathway in broilers. J Anim Sci, 97(4): 1679-1692, doi: $10.1093 /$ jas/skz078

Yongjun GHW, 2008. Effects of supplementation of dihydromyricetin in diet on intestine morphology, alkaline phosphatase and growth performace of broiler. China Feed, 6

Yue L, Li L, Zhang J-W, Zhong X-Q, Wei J-A et al., 2018. Total polysaccharides of the Sijunzi decoction attenuate tumor necrosis factor- $\alpha$ induced damage to the barrier function of a Caco2 cell monolayer via the nuclear factor-kB-myosin light chain kinase-myosin light chain pathway. World J Gastroenterol, 24(26): 2867-2877, doi: 10.3748/wjg.v24.i26.2867

Zhang B, Lv Z, Li Z, Wang W, Li Get al., 2018. Dietary L-arginine supplementation alleviates the intestinal injury and modulates the gut microbiota in broiler chickens challenged by Clostridium perfringens. Front Microbiol, 9: 1716, doi: 10.3389/fmicb.2018.01716

Zhao Y, Mao F, Zhao Q, Jing F and Jing K, 2015. Study on the anti inflammatory and anti gastric ulcer effect of Glycyrrhiza polysaccharide. AsiaPacific Trad Med, 11(9): 12-14

Zhu X, Xun LK and Zhang H, 2018. Study on antiinflammatory activity of Glycyrrhiza selenide. Heilongjiang Anim Husband Vet Med, 4:178-181

Zmora N, Levy M, Pevsner-Fishcer M and Elinav E, 2017. Inflammasomes and intestinal inflammation. Mucosal Immunol 10(4): 865-883, doi: $10.1038 / \mathrm{mi} .2017 .19$

Received - 16.07.2021, Accepted - 17.10.2021, Published - 01.12.2021

Section Editor: Dr. I. Samanta, Associate Editor 